\title{
A computer-oriented task analysis method for mathematics instruction
}

\author{
JOHN W. COTTON, SANDRA P. MARSHALL, and STANLEY VARNHAGEN \\ University of California, Santa Barbara, California 93106
}

and

\author{
JOHN P. GALLAGHER \\ Cleveland State University, Cleveland, Ohio 44102
}

\begin{abstract}
Components of a computer solution for fraction problems in arithmetic have an analog in a now approach to educational task analysis. The components may be called goal-setting or planning elements, in contrast to the goal-satisfying or behavioral steps emphasized in much contemporary task analysis. A hypothetical tutorial dialogue in which a student is asked to tell a tutor what step to perform next is presented as an example of the emphasis of the planning side of computation. Explicit identification of goal-setting elements of a task is illustrated by analysis of the task of converting a pair of fractions to a new pair with a common denominator. The decomposition thus produces is simulated with two computer programs: (1) a LISP program using a set of production rules consisting of conditions to be met and subsequent actions to be taken and (2) a PROLOG program stating goals and solving goals explicitly.
\end{abstract}

This article suggests a new method of task analysis for arithmetic and algebra. Two properties are desired for this method: (1) It should match certain intuitive notions about human solution of mathematics problems, and (2) it should yield a set of subtasks satisfying the logical requirements for solving the task. The intuitive notions involved are less weighty but are presented first to prepare the way for a more formal analysis closely related to computer theory.

We anticipate our argument by beginning with a very strong claim: The number of elements resulting

This article was prepared under Contract N001 23-77-C-0087 from the Navy Personnel Research and Development Center to the University of California, Santa Barbara (UCSB), a contract housed in the UCSB Computer System Laboratory and under Grant GRA-57954 from the Science Research Council to Alan Bundy and the Department of Artificial Intelligence, University of Iidinburgh. Appreciation is expressed to John Wolfe, Navy contract monitor, for his facilitation of and suggestions about this work, to James G. Greeno. Learning Research and Development Center, University of Pittsburgh, for consultations. and to Alan Bundy for his stimulation and hospitality during the period in which this article was completed. Lawrence Byrd of the Department of Artificial Intelligence, University of Idinburgh, made a major contribution to the PROLOG sections of this article; his aid is specially acknowledged here. John Bruno, Director of UCSB Computer Systems Laboratory, and James H. Block, Department of Education, UCSB, were helpful in their suggestions. The views and conclusions contained in this document are those of the authors and should not be interpreted as necessarily representing the policies, either implied or expressed, of the U.S. government or of anyone credited above with assistance. Reprints should be requested from John W. Cotton, Department of Fducation. Iniversity of Cilifornia. Santa Barbara, California 93106. from a decomposition of a mathematical task should be approximately twice as large as the number obtained in conventional task analysis using methods described by such authors as Gagné (1968) and White (1974). The ultimate reasons for this claim lie in the nature of mathematics itself. As later parts of this paper will claborate. every solution of a mathematical problem must include planning steps in addition to other steps commonly identified in educational task analyses. For example, the problem in Equation (1) requires more than the bare computational steps shown below for it:

$$
4 X+2 / 5=2 / 3 \text {. Solve for } X
$$

One method for solving this problem is as follows:

First, put the negative of the additive constant for the left-hand side of Equation 1 on each side of a revised equation, thus removing the original additive constant from the left-hand side and leaving its negative on the right:

$$
4 X=2 / 3-2 / 5 \text {. }
$$

Now convert the two fractions to new fractions with the same common denominator, yielding:

$$
4 X=10 / 15 \quad 6 / 15
$$

Third, subtract the second term, right-hand side, from the first term on that side, yielding:

$$
4 X=4 / 15 \text {. }
$$


Finally, divide through both sides of Equation 4 by the multiplier of $\mathrm{X}$, obtaining:

$$
X=1 / 15 \text {. }
$$

This sequence of responses may be viewed as one logical analysis of the total task into a temporal subsequence of specific computational steps. Notice that no attention was given to the problem of selecting which step to perform next. Much of the remainder of this paper will deal with such selection, to be called goal setting.

\section{METHODS FOR TRAINING GOAL SETTING}

How might a human tutor assist students to learn the procedures we call goal setting? One possibility is to teach by trial and error, with the option of providing hints from time to time. This approach might lead to the following conversation about another problem:

Tutor: Jerry is twice Bill's age. Jerry's age plus Bill's age is 24 . What is Jerry's age?

Student: I don't know how to begin.

Tutor: Why not assign letters to variables and try to state equations in place of the sentences in the problem?

Student: OK, I'll call Jerry's age $\mathrm{x}$ and Bill's age $\mathrm{y}$. Then $x=2 y$ replaces the first sentence, and $x+y=24$ replaces the second one. The third sentence means: find $x$.

Tutor: Fine. Now what needs to be done to solve the problem?

Student: Let's see-if I knew $\mathrm{x}$, I could find $\mathrm{y}$ with either equation. But how do I find $x$ ? I need more help.

Tutor: Well, one thing you could do is to substitute the symbolic value of $x$ in the first equation for $x$ in the second equation and see if the revised second equation is a help to you.

Student: I'll try that. Since $\mathrm{x}=2 \mathrm{y}$ in the first equation, the second equation's $x$ becomes $2 y$, and I get: $2 y+y=$

24 , or $3 y=24$. So $y=8$. Now I can go back to $x=2 y$ and find $x=2 \times 8=16$. Great! I think the method worked.

In such a conversation, the student would rely on the tutor for most goal setting. Thus the student was told to start by assigning letters to variables and to state equations in place of sentences. The student could successfully satisfy all goals once they were set. Could the total task be learned in this way? Yes, it seems reasonable that later tutorial conversations might lead the student spontaneously to propose goals to be set, with the tutor confirming or correcting such a response and thus serving to build up correct goal-setting responses.

How could this process be speeded up? A case can be made for the argument that the only new parts of a complex task of reasonable difficulty are its goal-setting components. If so, it might be useful to spend more time teaching the goal-setting than training the goal-satisfying parts of the task. This could be done by asking the student to tell the tutor what step to perform, having the tutor perform it, having the student tell the tutor the next step to perform, and so on. This procedure is illustrated in a further hypothetical dialogue:

Tutor: I want to find the value of $(1 / 2+1 / 3) \times 2 / 5$.

What should I do first?
Student: There are different ways to find the answer. Why don't you multiply $2 / 5$ by each term inside the parentheses?

Tutor: OK. That gives $(1 / 2 \times 2 / 5+1 / 3 \times 2 / 5)$, or $(2 / 10+$ $2 / 15)$. That simplifies to $(1 / 5+2 / 15)$. Now what?

Student: Well, why not multiply each fraction by 3 ? Tutor: I can't do that without changing the value of the expression.

Student: That's right. So let's do something else. Why not find two equivalent fractions with the smallest possible common denominator?

Tutor: Fine, that gives $(3 / 15+2 / 15)$, or $5 / 15$.

Student: Great. Now simplify that result.

Tutor: OK, that yields $1 / 3$.

Student: Now we're finished.

Tutor: Right on!

An alternate plan for the same problem is shown in the following conversation:

Tutor: I want to find the value of $(1 / 2+1 / 3) \times 2 / 5$. What should I do first?

Student: Why not simplify the problem first by combining terms inside the parentheses?

Tutor: OK, how do I do that?

Student: We need to add $1 / 2$ and $1 / 3$, so let's find their least common denominator.

Tutor: Fine, it's 6 . Now what?

Student: Let's convert $1 / 2$ to sixths and $1 / 3$ to sixths. Tutor: Then we have $3 / 6$ and $2 / 6$. What should I do next? Student: We were to add $1 / 2$ and $1 / 3$, so let's add $3 / 6$ and $2 / 6$.

Tutor: That sum is $5 / 6$. What do I do now?

Student: Well, the sum of $1 / 2$ and $1 / 3$ was to be multiplied by $2 / 5$. So let's multiply $5 / 6$ by $2 / 5$.

Tutor: OK, that yields $10 / 30$.

Student: Please simplify $10 / 30$.

Tutor: $1 / 3$.

Student: Fine. The problem is solved.

Tutor: I agree.

In the examples just presented, the student makes almost all decisions and the tutor acts as an intelligent computer (i.e., a computer that can accept a variety of instructions and act as requested but can refuse to perform improper tasks). Accordingly, we can view our proposed process of teaching goal setting as comparable to tutorial instruction in the programming of the mathematical task to be mastered. A good tutor can carry on his (her) side of both of the above dialogues because each dialogue is an intelligent one. An intelligent computer must function as well as human tutors do.

This sort of goal-setting training could be done by using a slight modification of a procedure employed in remedial mathematics computer-assisted instruction (CAI) system developed by Cotton, Gallagher, Hopkins, and Marshall (1978). Some students trained with that system are allowed the option of requesting computations of parts of problems. In the example of solving $(1 / 2+1 / 3) \times 2 / 5$, a student could type: COMPUTE $1 / 2+1 / 3$, COMPUTE $1 / 2 \times 2 / 5$, or COMPUTE $1 / 3 \times$ $2 / 5$ as an early step in the overall computation. Some modification of the COMPUTE command would be 
necessary to obtain dialogues similar to the one above. The tutorial procedure proposed here, if automated in a CAI system, would have a student give one COMPUTE command, wait for the result, and continue until the student concludes that the overall answer has been reached. Provided that the student's prerequisite skills in lower level computation were adequate before training of the complex task began, this method should lead to better performance on the total task than should an equivalent amount of training time on the task as a whole. We now examine possible computer program structures for representing mathematical skills like those we have been discussing.

\section{COMPUTER REPRESENTATIONS OF MATHEMATICAL PROBLEM SOLVING}

Two general kinds of computer programs tend to be employed in simulations of human reasoning: networks and production systems. Networks are sets of nodes connected by labeled arcs, permitting the retrieval of stored relationships and the deduction of relationships implied by what is stored. A production system is a set of if-then rules (production rules) stating conditions that, if met, lead to specified actions. Also, one or more rules are required to specify the order in which the condition-action rules are to be implemented.

Let us now consider a production system using the following operating procedure: (1) Try each production rule in sequence, beginning with the first. As soon as one is found whose conditions are met (both goal conditions and stored problem characteristics), do it. Then begin with the first and try each again until one can be performed. Continue in this way until no more rules can be applied. (2) When a goal is set, it preempts any earlier goal. (3) When a goal is satisfied, it is deactivated; the last goal preempted (if any) is reactivated.

So far, we have appealed to common sense to bolster the claim that solving mathematical problems consists of two kinds of subtasks: selecting computations to perform and performing them. We have called the former kind of subtask goal setting; it is now convenient to call the latter kind goal satisfying and to think of both kinds of subtasks as counterparts of the kinds of production rules just defined.

We can illustrate the proposed use of production systems in arithmetic by comparing such a system with the results of a more conventional analysis of a relatively simple task: converting two fractions to new fractions with a common denominator. A first look might suggest that only two subtasks are necessary to the total task: finding the least common denominator (LCD) and using that denominator to convert the original pair of fractions to a new pair with that denominator. However, other subtasks can easily be identified. In fact, it might be possible to continue subdivision of a task of this kind for many steps. Any subdivision we may suggest will be arbitrary, then. The only criteria we will set for such subdivisions are that the subtasks must be explicit enough to be performed by a machine and that they must be sufficient for the machine in question to complete the total task by performing those subtasks and no others. Table 1 presents a possible decomposition of the task. We believe this decomposition to satisfy the criteria just established, provided that the machine has Table 1 available and can read it. Note that satisfying these criteria is necessary but not sufficient for satisfying Gagné and Paradise's (1961, p. 4) criterion for identifying a prerequisite skill: "What would an individual have to know how to do in order to achieve successful performance of this class of tasks, assuming he were given only instructions?" Lower level prerequisites would also be found by the Gagne' and Paradise method.

The separate steps in Table 1 are all behavioral steps, in the sense that some directly observable response is required for each. These four steps represent the goalsatisfying parts of the production system we need.

What else is required by the student desiring to learn how to convert a pair of fractions to a new pair with the smallest possible denominator? The student must have the ability to tell what to do next (i.e., set the next appropriate subgoal). For example, the problem of Table 1 is to convert $(1 / 21 / 3)$ to a new fraction pair with the smallest possible common denominator. The initial task for the student faced with this problem is to recall the first appropriate subgoal, finding the LCD of $1 / 3$ and $1 / 2$. When three goal-setting subtasks are established (corresponding approximately to P7, P3, and P5 on the right-hand side of Table 2), one to match each subgoal, and added to the four goal-satisfying tasks of Table 1, we obtain the left side of Table 2, listing all subtasks of both kinds in the sequence in which they occur. Much of the task solution focuses on the third subtask, which forms a frame with blanks to be filled in later.

The right side of Table 2 presents relatively informal statements of seven production rules, P1-P7. They are arranged in the actual order a computer would call them to solve the same problem, given the operating procedure defined earlier for production systems. The right-hand side of the table parallels the left side fairly

Table 1

A Possible Behavioral Decomposition of the Task of Converting the Pair of Fractions, 1/3 and 1/2, to a New Pair With the Smallest Possible Common Denominator

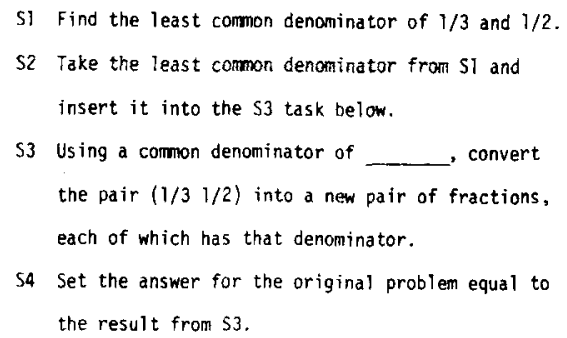


Table 2

Goal-Setting and Goal-Satisfying Steps in Conversion of a Fraction Pair

\begin{tabular}{|c|c|}
\hline $\begin{array}{l}\text { Recall: I must first find the least common } \\
\text { denominator of } 1 / 3 \text { and } 1 / 2 \text {. }\end{array}$ & $\begin{array}{l}\text { P7 Given goal (convert } 1 / 31 / 2 \text { ), set goal } \\
\text { (LCO } 1 / 31 / 2 \text { ). }\end{array}$ \\
\hline $\begin{array}{l}\text { Answer: What is the least common denomina- } \\
\text { tor of } 1 / 3 \text { and } 1 / 2 \text { ? } \\
\text { Recall: I must now move the answer from above } \\
\text { to the first blank of the statement below: }\end{array}$ & $\begin{array}{l}\text { P2 Given goals (LCO } 1 / 31 / 2 \text { ) and (Convert } 1 / 31 / 2 \text { ), } \\
\text { ask "What ts least common denominator of } 1 / 31 / 2 \text { ?" } \\
\text { Store answer in short term memory. Satisfy } \\
\text { (LCD } 1 / 31 / 2 \text { ). }\end{array}$ \\
\hline $\begin{array}{l}\text { Using a cormon denominator of } \\
\text { leads to the transformation of the pair } \\
1 / 31 / 2 \text { to a new pair with values }\end{array}$ & $\begin{array}{l}\text { P3 Given fact (least common denominator } 1 / 31 / 2=6 \text { ), } \\
\text { mark the fact for no further use and set goal } \\
\text { (conversion-LCD-value } 1 / 31 / 2 \text { ). }\end{array}$ \\
\hline and ___ respectively. & P4 Given goal (conversion-LCD-value $1 / 31 / 2$ ), set \\
\hline & goal (conversion 6) and satisfy (conversion- $L C O$-value). \\
\hline paragraph. & \\
\hline $\begin{array}{l}\text { Recall: I must now perform the transfor- } \\
\text { mation required to fill in the last two } \\
\text { blanks of the earlier paragraph. }\end{array}$ & $\begin{array}{l}\text { P5 Given goals (conversion } 6 \text { ) and (convert } \overline{1 / 3} 1 / 2 \text { ), } \\
\text { set goal (transform } 1 / 31 / 26 \text { ) and satisfy (conver- } \\
\text { ston } 6 \text { ). }\end{array}$ \\
\hline $\begin{array}{l}\text { Perform the transformation just mentioned } \\
\text { and thus fill in the last two blanks of the } \\
\text { earlier paragraph. }\end{array}$ & $\begin{array}{l}\text { P6 Given godl (trans form } 1 / 31 / 26 \text { ), ask "What is } \\
\text { conversion } 1 / 31 / 26 \text { ?" Store the answer in short term } \\
\text { metrory. Satisfy goal (transform } 1 / 31 / 26 \text { ). }\end{array}$ \\
\hline $\begin{array}{l}\text { Set the problem's answer equal to the } \\
\text { result of the transformation just finished. }\end{array}$ & $\begin{array}{l}\text { P1 Given fact (conversion } 1 / 3 \quad 1 / 26=2 / 63 / 6 \text { ) and } \\
\text { goal (convert } 1 / 31 / 2 \text { ), output: "Answer is } 2 / 63 / 6 . " \\
\text { Mark all renaining items in short term menory so that } \\
\text { the production system will stop. }\end{array}$ \\
\hline
\end{tabular}

Note-The problem shown is to convert $(1 / 31 / 2)$ to a new fraction pair with the smallest possible denominator. Psychological steps are on the left. Comparable production rules are on the right.

closely, showing that a machine can indeed simulate the processes we are assuming to operate with humans. Notice that, because the focus of this section is on the strategy of solving a problem, this production system is not a simulation of all calculations used in solving the problem. Rather, it shows successive high-level stages presumed operative in humans, letting the questions and answers in $\mathrm{P} 2$ and $\mathrm{P} 4$ produce the results of low-level calculations. This way of displaying processes of interest and ignoring others is comparable to Anderson's (1976, pp. 203-204) calling of as-yet-undefined subroutines for one of his production systems.

The reader is referred to Appendix A for a discussion of our seven rules in the exact form in which they have run on a PDP-11/45 computer, using a slightly adapted version of Winston's (1977, pp. 364-366) production system interpreter. Appendix A also includes a trace of the results of each successful call of a production rule during the solution process.

A second computer representation of the task at hand uses a language, PROLOG, in which the distinction between goal setting and goal satisfying is more explicit than in LISP networks or in production systems. Appendix B presents an example of the use of PROLOG to set and to satisfy the goals into which our fractionconverting task has already been analyzed. Both a set of relevant programs and a trace of their operation are presented in Appendix B.

The PROLOG program of Appendix B has the same basic components as the production system of Table Al but is a quite different program and thus a different psychological theory. Table B1 shows that the new solution process begins by subdivision of the "convert" goal into the subgoals "Icd" and "conversion." After solution of the first subgoal, "conversion" (corresponding to "conversion6" in Table A1) is subdivided into "conversion_LCD_value" and "transform." Each of these subgoals is performed in turn. Thus, this program is the solution of an AND-tree, and it accomplishes its task by solution of its subproblems in proper order. In contrast, the production system of Table Al often sets one subgoal at a time. One might view the PROLOG program as the behavior of a person faced with a fraction-conversion task for the first time, leading to an explicit backward inference method of decomposing each goal or subgoal into its parts before trying to solve any subgoal. Correspondingly, one might view the production system as an algorithm generated by a person having prior knowledge of the goal structure. Or the production system may be loosely viewed as a depthfirst specification of solution steps in the AND-tree just mentioned. 


\section{EDUCATIONAL IMPLICATIONS OF TABLES 1 AND 2}

Notice that, whereas Table 1 used the behavioral components of conventional task analysis, Table 2 almost doubled the number of components employed by associating a task recall (goal-setting) component with each original behavioral subgoal. Thus an information processing theory approach to instruction has greatly increased the number of task elements to be taught.

We believe that this emphasis upon teaching both goal-setting and goal-satisfying elements, if followed, would force much more attention to some usually covert behaviors of students. The present approach suggests that much more attention should be given to training the planning, cognitive, or goal-setting components of a task being taught.

One difficulty that arises immediately is that some means of making internal responses overt is required during training. Those responses are often suppressed by teachers and employers who wish to focus upon end products rather than upon the processes involved in mathematical work. This difficulty is compounded by the fact that increased observation by the teacher is desirable and may even require a one-to-one teachinglearning relationship because of the importance of helping the student to recognize an unwise plan. Making the responses overt permits direct reinforcement of them, something impossible otherwise. But it certainly may burden the teacher by doubling the detail with which work on a given problem needs to be analyzed; now one must look at goal-setting operations as well as conventional computational responses.

The tutorial dialogue presented earlier as a way of teaching the goal-setting operations for solving $(1 / 2+1 / 3) \times 2 / 5$ may be ideal, but few school boards can give financial support to regular dialogues of this kind using human teachers and students in one-to-one relationships. Accordingly, CAI approaches to teaching are potentially more practical than is increased use of teachers for the training of goal-setting behavior.

The learning of multielement tasks such as those we have been discussing may be formally represented either by hierarchical or nonhierarchical Markov models (Cotton, Marshall, Varnhagen, \& Gallagher, 1979) or by adaptive production systems, production systems with the capability to modify themselves appropriately in response to learning experiences (Waterman, 1975; Waterman \& Hayes-Roth, 1978).

The computational model developed above has similarities to one of three models developed by Greeno (1976) for solving problems such as $2 / 5=x / 15$. His third method is a computational algorithm much like our production system for converting two fractions. Although Greeno represented it in flowchart form, he could easily have displayed it as a LISP program or as a production system.

Greeno's (1976) first and second methods are cognitively richer than the algorithmic approaches just discussed, because they treat numbers as representing quantities rather than just as symbols to be manipulated. Method 1 is geometric, generating equivalent fractions with different denominators as different partitionings of the same portion of a unit-sized region. Method 2 is similar, except that it employs appropriate numbers of elements and subsets of a set. Use of words and pictures in cognitive models for mathematics instruction is likely to facilitate learning the geometric, set theory, and real-life meanings of concepts such as fractions.

\section{CONCLUSION}

The foregoing remarks leave us with a somewhat novel approach to task analysis, which now needs to be placed in historical perspective. Task analysis may be performed for at least three purposes: (1) to facilitate training, (2) to facilitate supervision of already trained workers, and (3) to facilitate understanding of the processes of complex behavior. These functions of task analysis receive varying degrees of emphasis in reviews by Davies (1973), Duncan (1972), Resnick (1976), and Gibbons (Note 1).

A common approach to training has been outlined by Pease and Lambert (1977), who recommend four steps. First, decide what skill is to be taught. Second, define the task operationally. Third, arrange the sequence of subtasks to be taught. Finally, use a teaching program with remedial loops to ensure learning of subtasks when learning is initially slow. As mentioned earlier, Gagné and Paradise (1961, p. 4) have posed a question that can be used for identification of one or more subtasks prerequisite to a high-level task: "What would an individual have to know in order to achieve successful performance of this class of tasks, assuming he were given only instructions?" Repeated use of the question to identify the prerequisites of other prerequisite subtasks allows development of a substantial structure of knowledge, with a preferred order of teaching its components.

Examination of hierarchies of knowledge developed by Gagné and his associates shows great reliance on behavioral subtasks, as might be expected from the emphasis on operational task definition made by scholars such as Pease and Lambert (1977). However, Gagné and Paradise's (1961) use of the term "know" leaves room for the identification of cognitive rather than behavioral subtasks.

It may be noted that cognitive psychology commonly employs theoretical concepts to explain overt behavior. Sometimes, as in the case of Rundus and Atkinson's (1970) research on rehearsal in free recall, procedures are devised to make these theoretical processes observ- 
able. In other cases, it seems difficult or impossible to do so. Suppes' (1973) use of "carry" and "no-carry" states in a model for arithmetic addition is a further cognitive example of a theoretical analysis with behavioral implications.

In a very extensive task analysis of introductory mathematics, Resnick, Wang, and Kaplan (1973) have developed hierarchies of high-level behavioral objectives. For example, consider their Objective 1-2:E, to count out a subset of $n$ objects from a set of movable objects, moving them out of the set as they are counted. The Resnick et al. (1973, p.687, p. 690) analysis of this objective has the child "store" the numeral (Box 11a in their Figure 6), remember the numeral while counting (Box IlIb in their Figure 6), and (implicitly) recognize when the stored numeral is reached in order to stop counting (Box IIc in their Figure 6). Similar cognitive steps are identified in the Resnick et al. Objectives 1$2: F, 5: A, 5: B, 5: E$, and $6: B$.

Like Resnick et al. (1973) and Suppes (1973), the present article employs both cognitive and behavioral concepts in its task analysis. A temporal hierarchy is implied by the analysis of Table 2 above. However, we have not sought experimental evidence that it or any other hierarchy represents a set of organized prerequisites for the overall task of converting two fractions to a new pair with a common denominator. An earlier paper (Cotton, Gallagher, \& Marshall, 1977) indicated that identification and validation of such prerequisite hierarchies are more difficult than is commonly reported.

The present article may also be distinguished from earlier cognitive task analyses in its pairing of cognitive (goal-setting) and behavioral (goal-satisfying) components of a problem. Finally, we remark that use of a computer simulation of task solution reflects the logical requirement that a human must know as much as a computer would need to "know" to perform the same task.

\section{REFERENCE NOTES}

1. Gibbons, A. S. A review of content and task analysis methodology (Tech. Rep. No. 2). San Diego: Courseware, Inc., March 1977.

2. Pereira, L. M., Pereira, F. C. N., \& Warren, D. H. D. User's guide to DEC system 10 PROLOG (Occasional Paper No. 15). Edinburgh: University of Edinburgh, Department of Artificial Intelligence, September 1979.

3. Byrd, L. PROLOG debugging facilities (Occasional Paper No. 19, Part 2). Edinburgh: University of Edinburgh, Department of Artificial Intelligence, July 1980.

4. Byrd, L., Pereira, F., \& Warren, D. A guide to Version 3 of DEC-10 PROLOG (Occasional Paper No. 19, Part 1). Edinburgh: University of Edinburgh, Department of Artificial Intelligence, July 1980.

\section{REFERENCES}

Anderson, J. R. Language, memory, and thought. Hillsdale, N.J: Erlbaum, 1976.

Cotton, J. W., Gallagher, J. P., Hopkins, M., \& Marshall, S. P. A multimethod generative CAI system for remedial mathematics. Behavior Research Methods \& Instrumentation, 1978, 10, 203-212.
Cotton, J. W., Gallaghen, J. P., \& Marghall, S. P. The identification and decomposition of hierarchical tasks. American Educational Research Journal, 1977, 14, 189-212.

Cotton, J. W., Marshall, S. P., Varnhagen, S., \& Gallagher, J. P. Theory of learning mathematics with computer-assisted instruction: Hierarchical and nonhierarchical options. Behavior Research Methods \& Instrumentation, 1979, 11, 139-146.

Davies, I. K. Task analysis: Some process and content concern. AV Communications Review, 1973, 21, 73-86.

Duncan, K. Strategies for analysis of the task. In J. Hartley (Ed.), Strategies for programmed instruction: An educational technology. London: Butterworths, 1972.

GaGné, R. M. Learning hierarchies. Educational Psychologist, $1968,6,1-9$.

Gaqné, R. M., \& Paradige, N. E. Abilities and learning sets in knowledge acquisition. Psychological Monographs, 1961, $75(14$, Serial No. 518$)$.

GreEno, J. G. Cognitive objectives of instruction: Theory of knowledge for solving problems and answering questions. In D. Klahr (Ed.), Cognition and instruction. Hillsdale, N.J: Erlbaum, 1976.

Kowalsxi, R. Logic for problem solving. New York: North Holland, 1979.

McDermott, D. The PROLOG phenomenon. ACM SIGART Newsletter, 1980, 72, 16-20.

Pease, D., \& Lambert, J. A task analytic approach to designing curricula for the behaviorally retarded. Psychologica Belgica, $1977,17,157-164$.

Resnick, L. B. Task analysis in instructional design: Some cases from mathematics. In D. Klahr (Ed.), Cognition and instruction. Hillsdale, N.J: Erlbaum, 1976.

Resnick, L. B., Wang, M. C., \& Kaplan, J. Task analysis in curriculum design: A hierarchically sequenced introductory mathematics curriculum. Journal of Applied Behavior Analysis, $1973,6,697-710$.

Rousser, P. PROLOG: Manuel de reference et d'utilisation. Luminy: Universite d'Aix-Marseille, Group d'intelligence Artificielle, 1975.

Rundus, D., \& Atrinson, R. C. Rehearsal processes in free recall: A procedure for direct observation. Journal of Verbal Learning and Verbal Behavior, 1970, 9, 99-105.

Suppes, P. Facts and fantasies of education. In M. C. Wittrock (Ed.), Changing education: Alternatives from educational research. Englewood Cliffs, N.J: Prentice-Hall, 1973.

Waterman, D. A. Adaptive production systems. In Fourth International Joint Conference on Artificial Intelligence. Proceedings. Cambridge, Mass: M.I.T, Artificial Intelligence Laboratory, 1975.

Waterman, D. A., \& Hayes-Roth, F. (EDs.). Pattern-directed inference systems. New York: Academic Press, 1978.

WhITE, R. T. The validation of a learning hierarchy. American Educational Research Journal, 1974, 11, 121-136.

Winston, P. H. Artificial intelligence. Reading, Mass: AddisonWesley, 1977.

\section{APPENDIX A \\ A PRODUCTION SYSTEM FOR CONVERTING FRACTION PAIRS}

The main body of this article includes an informal listing of seven production rules for converting a fraction pair to a new fraction pair with a common denominator. Table 2 showed a parallelism between those rules and hypothesized psychological events in the solution of such problems. We now present the same production system in a form that has actually been run on a PDP-11/45 computer employing a UNIX operating system and a slightly modified version of Harvard LISP.

Since the key to this computer run is Winston's (1977, pp. 364-366) interpreter for production systems, we begin with some remarks about it. This interpreter is a LISP program for 
Table A I

A Production System for Converting a Fraction Pair to a New Pair With a Common Denominator

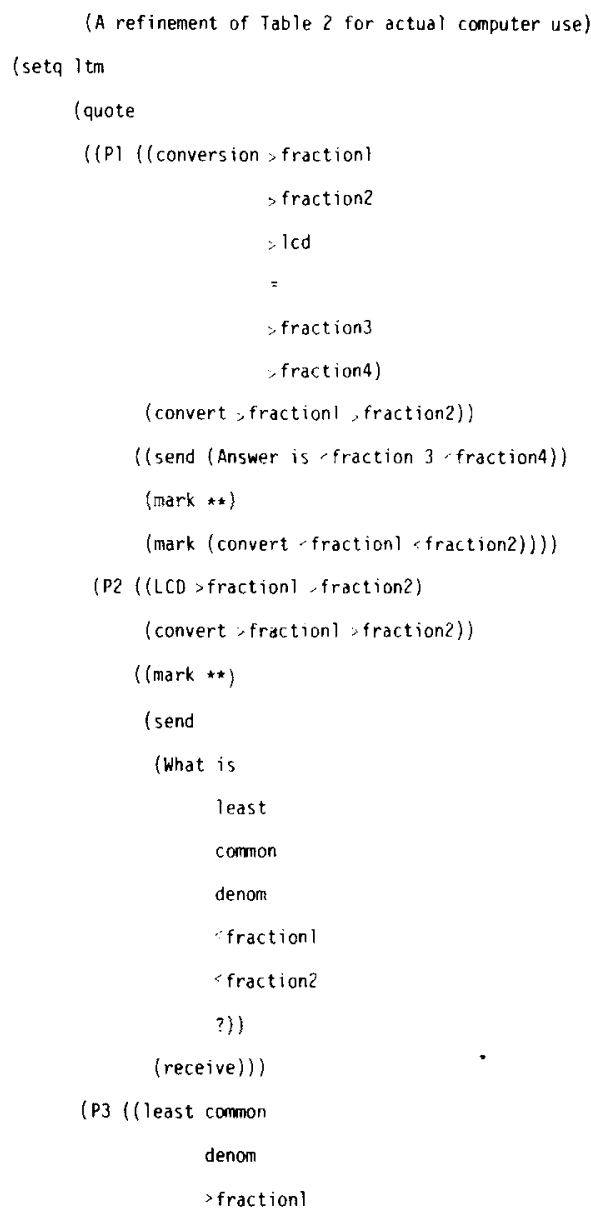

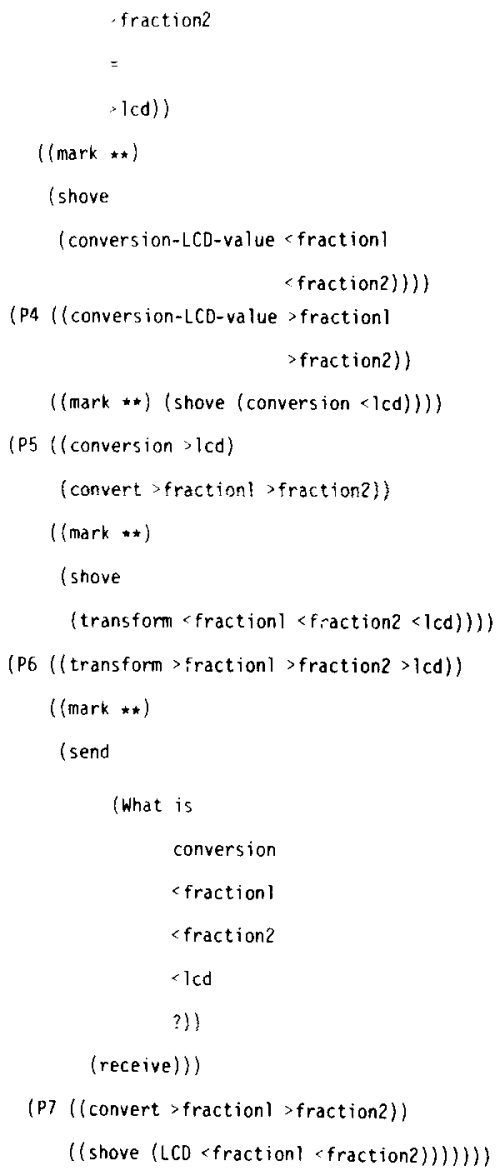

examining production rules sequentially, executing the action side of the first rule for which the condition(s) set by the rule is (are) met, as seen by comparison with the current short-term memory (STM) of the system. Execution of a rule leads to modification of STM, after which examination of rules begins again with the first production. The process continues until no further acts can be generated.

Four kinds of actions are of interest here: (1) a shove command puts a new goal or fact into the left-hand side of STM and deletes its rightmost member so as to keep the number of entries constant; (2) a send command asks another computer program or the computer user for information; (3) a receive command accepts information given in response to send; and (4) a mark command labels an STM entry with the word "mark" in order to remove its power to satisfy conditions for a production. The mark command may either be written (mark**), to say that the first item in STM is to be marked, or be written (mark clause), where a specific expression replaces "clause" so that the second or later item of STM can be marked.

For the present paper, Winston's (1977) interpreter has been slightly modified. It now can load STM and long-term memory (LTM, which for this model is the set of productions). It can also display the initial STM entries and (when desired) each successive new version of STM. Further minor modification was done so that it would run with the LISP dialect already mentioned as being employed.
Table A1 presents the refinement of the Table 2 production system used with the Winston (1977) interpreter. Production names P1-P7 permit comparison with corresponding rules of Table 2. Table A1 is simply a LISP setq instruction setting LTM to a list of all seven production rules.

Notice that the condition(s) set by a production follow(s) a label such as $\mathrm{PI}$, each condition being enclosed in parentheses and the entire set of conditions being further enclosed. For example, the single condition for $\mathrm{P} 4$ is (conversion-LCD-value $>$ fraction $1>$ fraction 2), meaning that a goal of finding a conversion based on a pair of fractions has been stored. The symbol ">" means that any value of the variable following the symbol will be accepted.

Similarly, the action(s) to be taken once the conditions of a production have been met is (are) shown in the last half of the production. Again, parentheses surround individual actions, and the total set of actions is surrounded by parentheses. Thus two actions are used in P4: (mark**), as previously defined, and (shove (conversion < lcd)), which means to perform the shove operation on the conversion goal for a specific LCD. The < sign means that the variable following it has a specific value bound to it that must be used here.

Figure $1 \mathrm{~A}$ includes two flowcharts, with the left one showing the use of the production system interpreter with the present example. As this figure indicates, a run command in the first box leads to a search through all seven productions, with P7 


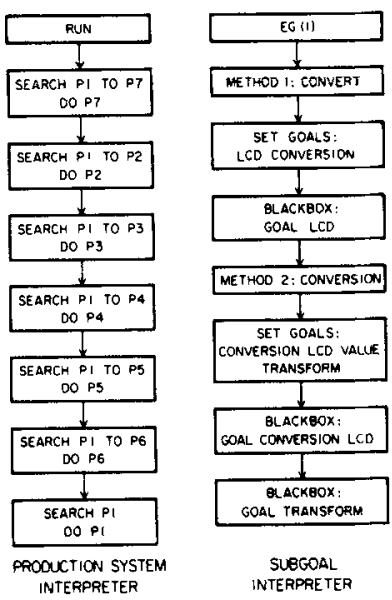

Figure Al. Flowcharts of the operation of the production system interpreter (left) and subgoal interpreter (right) in performing the fraction-converting task of Table 2. being the first whose conditions are met (second box). So the action part of P7 is performed. The next box in the flowchart shows that a new search begins with $P 1$ and stops with $P 2$, since its conditions are met, leading to performance of the action part of P2. Successive boxes show further searches and actions, with the final box leading to performance of $\mathrm{P} 1$; no further actions are possible thereafter.

Table A2 below is the trace given by the production system interpreter when LTM has the value in Table A1, STM initially has the value shown near the top of Table A2, and the run command given to the computer includes a trace flag.

As may be seen by comparison of Tables A1 and A2, STM originally did not meet the conditions for any production except P7. This led to a new STM:

$$
\text { ((LCD } 1 / 31 / 2=6) \text { (convert } 1 / 31 / 2) \text { nil nil nil nil nil), }
$$

which did not meet the conditions of P1 but did meet those of P2. After the actions of P2 were performed, the process continued with still another value of STM. The final stage of Table A2 occurred when P1 operated, with one of its consequences being the marking of all currently active goals, making further operation of the production system interpreter impossible.

Table A2

A Trace of the Operation of the Production System of Table A1

stm $=(($ convert "1/3" "1/2") nit nil nil nil nil nil)

( (LCD " $1 / 3 " ~ " 1 / 2 ")$

(convert " $1 / 3 "$ "1/2")

nil

nil

nil

nil

ni1)

\> What is least common denom $1 / 31 / 2$ ?

* least common denom " $1 / 3$ " " $1 / 2 "$ = "6"

(( east common denom "1/3" "1/2" = "6")

(mark (LCD "1/3" " $1 / 2 ")$ )

(convert "1/3" "1/2")

nil

nil

nii)

((conversion-LCD-value "1/3" "1/2")

(mark (least common denom "1/3" "1/2" = "6"))

(mark LCD "1/3" "1/2"))

(convert "1/3" " $1 / 2 "$ )

nil

nโ1

nil)

\footnotetext{
((conversion "6")

(mark (conversion-LCD-value "1/3" "1/2"))

(mark (least common denom "1/3" "1/2" = "6"))

(mark (LCD "1/3" "1/2"))
}

(convert " $1 / 3$ " " $1 / 2 "$ )

n11

niT)

((transform "1/3" "1/2" "6")

(mark (conversion "6"))

(convert " $1 / 3 " 1 / 2 "$ )

(mark (conversion-LCD-vaiue "1/3" "1/2"))

(mark (least common denom "1/3" "1/2" = "6"))

(mark (LCD "1/3" "1/2"))

nil)

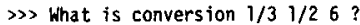

- conversion " $1 / 3$ " "1/2" "6" = "2/6" "3/6"

( (conyersion ${ }^{n} 1 / 3^{n} " 1 / 2^{n} " 6^{n}=" 2 / 6^{n}{ }^{n} 3 / 6^{n}$ )

(mark (transform "1/3" "1/2" "6"))

(mark (conversion "6"))

(convert " $1 / 3$ " "1/2")

(mark (conversion-LCD-value "1/3" "1/2"))

(mark (least common denom "1/3" "1/2" = "6"))

(mark (LCD "1/3" "1/2")))

$\gg$ Answer is $2 / 63 / 6$

((mark (convert " $1 / 3$ " "1/2"))

(mark (conversion "1/3" "1/2" "6" = "2/6" "3/6"))

(mark (transform " $1 / 3$ "

(mark (conversion "6"))

(mark (conversion-LCD-value "1/3" "1/2"))

(mark (least common denom "1/3" "1/2" = "6"))

(mark (LCD "1/3" "1/2"))) 
One advantage of using production systems for the modeling of cognitive processes is the transportability of such models from one computer to another. Use of different LISP dialects will require use of slightly different versions of Winston's (1977, pp. 364-366) production system interpreter. But a production system such as that given in Table A1 has a production system syntax and is almost completely dialect independent, enabling investigators at different places to test it locally if they have an appropriate interpreter available.

\section{APPENDIX B}

This appendix presents a second computer representation of the mathematical task components of Tables 1 and 2, using the PROLOG (programming logic) language of Roussel (1975), as implemented on the PDP-10 computer by Pereira, Pereira, and Warren (Note 2). Other sources of information about this language and its use appear in Kowalski (1979), McDermott (1980), Byrd (Note 3), and Byrd, Pereira, and Warren (Note 4).

We first performed the task at hand without a separate interpreter, workirg directly with the goal-setting (head) and goal-satisfying (body) forms of PROLOG clauses, corresponding closely to the Horn clauses of predicate calculus. However, a more elegant program has been developed with the aid of Lawrence Byrd of the Department of Artificial Intelligence, University of Edinburgh, who built a simple subgoaling interpreter with two pages of code, in order to have a more orderly data base for the problem, and did much of the programming in Table B1.

Table B1 contains two programs, METHOD and STUFF. The former contains two clauses labeled "Methods" and three labeled "Blackboxes," since they deal with subprocesses that were hidden from the reader of the LISP program of Table A1, being evoked there by interrogation of the program user. METHOD also contains a clause, "eg(1)," for starting the computation process with a certain example. STUFF contains clauses for finding the LCD via the least common multiple (LCM) and greatest common divisor (GCD) and for finding the mapping from one pair of fractions to another once the LCD of the first is known. Comment sections are made in two ways, either with $\%$ at the beginning or with $/ *$ at the beginning and * / at the end of the comment. An almost identical PROLOG program runs under UNIX with the PDP-11/45.

Turning back to the righthand side of Figure Al allows us to see the flow of control of the subgoal interpreter using the processes of Table Bl. Once eg(1) has been called (top box of Figure A1), starting the process by setting the goal of converting the pair $(1 / 2,1 / 3)$ to a new pair with a common denominator, the first method clause is called (second box) because its goal is "convert." That clause sets two subgoals, "LCD" and "conversion," shown in the third box. The first can be solved by the first blackbox, but the second requires a call of the second method. In turn, this method sets two subgoals, "conversion_LCD_value" and "transform," each of which can be solved by a blackbox.

The role of the blackboxes deserves a bit of further attention: On one hand, they represent processes that are not as carefully analyzed into goals and subgoals as the items in the methods clauses. This reflects a customary scientific focus upon certain aspects of a process, to the exclusion of others of less current interest. They are simply the engines for performing the goal-satisfaction tasks set in the methods. On the other hand they reflect some of the advantages of PROLOG over Harvard LISP. On one hand, the "find_LCD" operation called by the

Table B 1

PROLOG Programs Titled METHOD and STUFF Used With a Subgoal Interpreter in Converting a Fraction Pair to a New Pair With a Common Denominator

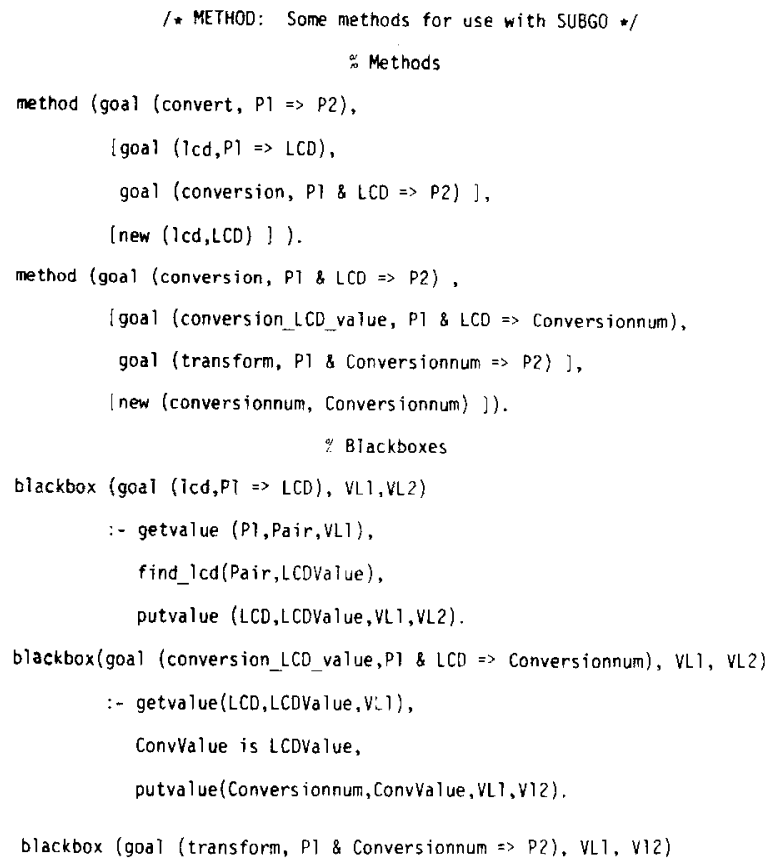


first blackbox is simpler than that in LISP. However, the big advantage of PROLOG, and the one that allowed display of the blackboxes rather than queries to program users, as in Tables Al and A2 with LISP, is that the writing of fractions is much easier with PROLOG.
The course of operation of the program of Table B1 is shown in Table B2, an actual trace of a program run. This trace shows the updating of two stacks, one for goals and the other for known and desired parameter values. Whenever a goal is solved, that fact is recorded on the trace.

Table B2

A Trace of the Operation of the Programs of Table B1

i ?- eg(1),

Running...

GOALS

$$
\text { goal (convert, } \mathrm{p} \text { ? } \Rightarrow \text { ans) }
$$

VLIST

value $(p 1$, pair $(1 / 2,1 / 3))$

value(ans, unknown)

goal (convert, $p$ l $\Rightarrow$ ans) solved by method

GOALS

$$
\text { goal }(1 c d, p|\Rightarrow| c d 1)
$$

goal (convers ion, pls1cdl $\Rightarrow$ ans)

VLIST

value (1cdl, unknown)

value (pl,pair $(1 / 2,1 / 3))$

value (ans, unknown)

goal ( Ted,pl=> led]) solved by blackbox

GOALS

goal (convers ion, $p 181$ ledl $\Rightarrow$ ans)

VLIST

value $(1 c d \mid, 6)$

value(pl,pair(1/2,1/3))

value(ans, unknown)

goal (conversion, $p|\&| c d l=>$ ans) solved by method

GOALS

goal (conversion_LCD_value,pl\&1cdl =>convers ionnuml)

goal ( transform, pl \&conversionnuml =>ans)
VLIST

value (convers ionnuml, unknown)

value $(1 \mathrm{cdl}, 6)$

value $(p 1, p a i r(1 / 2,1 / 3))$

value(ans, unknown)

goal (conversion_LCD_value, p1\&Icdl=>conversionnuml) solved by blackbox GOALS

goa) ( trans form, placonversionnuml $=>$ ans)

VLIST

value (convers ionnuml, 6)

value $(\operatorname{lcd} 1,5)$

value $\{p l, p a i r(1 / 2,1 / 3))$

value (ans, unknown)

goa 1 (transform, pl\&conversionnuml=>ans) solved by blackbox

GOALS

VLIST

value(ans, pair $(3 / 6,2 / 6))$

value (convers ionnuml, 6 )

value $(1 \mathrm{~cd} 1,6)$

value(pl,pair(1/2,1/3))

No more goals

VLIST

value(ans, pair $(3 / 6,2 / 6))$

value (conversionnuml, 6)

value $(1 c d T, 6)$

value $(p 1$, pair $(1 / 2,1 / 3))$ 